\title{
Bounding Demand Paging Costs in Fixed Priority Real-Time Systems
}

\author{
Young-Ho Lee ${ }^{1}$, Hoyoung Hwang ${ }^{2}$, Kanghee Kim ${ }^{3}$, and Sung-Soo Lim ${ }^{1}$ \\ ${ }^{1}$ School of Computer Engineering, Kookmin University, \\ Jungrung 3-dong, Seongbuk-gu, \\ Seoul, Korea 136-702 \\ \{buriburi, sslim\}@kookmin.ac.kr \\ ${ }^{2}$ Department of Multimdia Engineering, Hansung University, \\ Samsun-Dong 3Ga 389, Sungbook-Gu, \\ Seoul, Korea 136-792 \\ hyhwang@hansung.ac.kr \\ ${ }^{3}$ Mobile Communication Division, \\ Telecommunication Network Business, \\ Samsung Electronics Co., LTD. \\ Kang.hee.kimesamsung. com
}

\begin{abstract}
In real-time systems, demand paging has not been used since worst case performance analysis technique for demand paging is not available. In this paper, we propose a technique to obtain the worst case bound on the demand paging costs and devise a worst case response time analysis framework considering the demand paging costs. We divide the technique into two different methods, DP-Pessimistic and DP-Accurate, depending on the accuracy and the complexity of analysis. DP-Accurate considers the page reuses among task instances and thus gives more accurate analysis results. We evaluate the accuracy of the proposed techniques comparing the analysis results from DP-Accurate and DP-Pessimistic.
\end{abstract}

Keywords: WCET analysis, WCRT analysis, worst case response time, demand paging, flash memory.

\section{Introduction}

Demand paging has long been the key feature of virtual memory systems as fullfeatured operating systems are increasingly used in various computing devices. Until recently, the demand paging technique has been mainly used in desktop or server systems rather than real-time embedded systems. This is due to the fact that the applications in real-time embedded systems have not been such heavy-weighted ones that the demand paging do not have to be adapted in the systems. Therefore, rather than using demand paging, the whole program image is copied into DRAM before execution (we call it shadowing). The shadowing has not been considered as preferable because of its DRAM consumption.

With the advent of multi-functional and high performance real-time embedded systems, there is no choice but to use demand paging in the operating systems for 
real-time embedded systems. One major factor that makes adapting the demand paging in real-time embedded systems difficult is the unpredictable performance of demand paging. The unpredictability stems from the history-sensitive feature of demand paging that the page fault delay would be highly dependent upon the past memory access patterns. Though such difficulty exists, we would need any framework to analyze the demand paging performance if the demand paging is to be used in realtime system design.

In this paper, we devise a worst case response time (WCRT) analysis technique considering the demand paging cost in fixed priority scheduling real-time systems. The basic idea is that the conventional WCRT analysis framework is augmented with the calculation of additional cost caused by demand paging. One simple solution for the analysis would be deriving the worst case page fault cost for a whole task and add the cost to the original response time of the task. Such naïve solution would yield too loose bound on the WCRT considering demand paging cost. Therefore, we enhance the accuracy of the analysis by considering the possibility of page reuses among the task instances. We propose graph theoretical formulation for the analysis considering page reuses among the task instances. We show that the proposed enhanced technique produces more accurate bounds on the worst case demand paging cost than the simple and naïve technique up to $57 \%$ by simulation. The rest of this paper is organized as follows. Section 2 describes our WCRT analysis method. Section 3 shows the simulation results for our WCRT analysis method with a simple simulator and virtual task sets. Finally, we conclude in section 4.

\section{Bounding Demand Paging Cost}

In this section, we provide how we extend the original WCRT analysis to consider the additional costs of demand paging. Our technique is based on the conventional WCRT analysis frameworks[1][2][3][4][5][6] and an extension of the original framework to consider cache-related preemption costs[5]. In [5], the WCRT of task $\tau_{i}$ is defined as follows:

$$
R_{i}=C_{i}+\sum_{j \in h p(i)}\left\lceil\frac{R_{i}}{T_{j}}\right\rceil C_{j}+P C_{i}\left(R_{i}\right)
$$

where $P C_{i}\left(R_{i}\right)$ is the total preemption costs of task $\tau_{i}$. In this paper, we only focus on how the page fault handling cost could be considered in WCRT analysis leaving the consideration of remaining factors affecting the accuracy of the analysis for future work.

For simplicity of our analysis, we make the following assumptions: First, we assume the page fault handling cost for each page is identical in all cases. Second, we assume that our target system has infinitive memory and thus our target system does not suffer from memory limitation causing page replacement. The second assumption is to exclude the worst case page replacement analysis issue from the scope of the paper while the replacement policy should ultimately be considered for completeness.

The key issue of the WCRT analysis for demand paging is how to obtain the demand paging cost for each task and how to take into account it in computing the worst case response time of a task. In order to obtain the demand paging cost for each task, 
we introduce two analysis steps: demand paging cost analysis and page reuses aspect analysis. In the demand paging cost analysis step, the demand paging costs for every execution path is calculated. For an execution path-wise analysis, we use control-flow based approach. After the analysis, we obtain the demand paging cost and pages to be loaded for every execution path of the task. In the page reuses aspect analysis step, the page reuses among task instances are analyzed and we obtain the WCETs of each task instance considering the demand paging cost and the page reuses. We use graphtheoretical formulation to derive the worst case bound on the page fault cost of a series of task instances.

\subsection{Demand Paging Cost Analysis}

The objective of the demand paging cost analysis is to obtain the demand paging cost for every execution path. Because the demand paging cost is highly dependent on the execution paths of the task, we use control flow-based approach to obtain the demand paging cost depending on the execution paths. The first step of building the control flow graph for our analysis is to make the correspondence between each basic block and each memory page. All the basic block nodes in the control flow graph are mapped to the corresponding memory pages. Since the sizes of basic blocks are various, the page boundaries do not always match basic block boundaries. Figure 1 shows an example of basic block and page mapping for a sample control flow graph. The control flow graph has four different execution paths and each basic block is mapped to one of the six different pages.

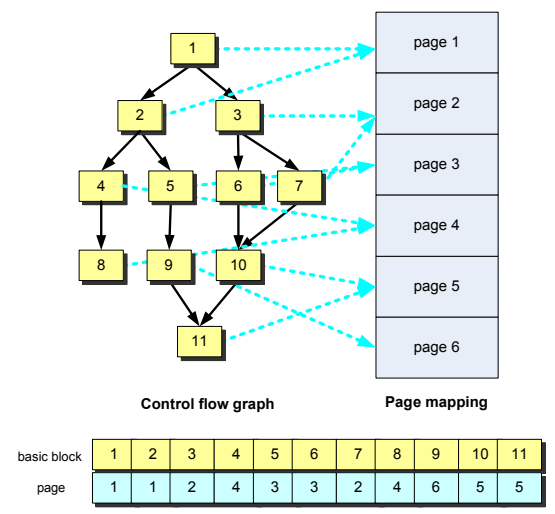

Fig. 1. Basic block and page mapping for a control flow graph

We use the following notations for formulation of our analysis: $C_{i, j}$ denotes the WCET of $j$-th execution path of task $\tau_{i}$. Note that $C_{i, j}$ is the pure WCET which does not consider the demand paging cost. $P_{i, j}$ denotes the set of pages for $i$-th execution path of task $\tau_{i}$, and $n\left(P_{i, j}\right)$ denotes the number of pages in $P_{i, j} . \pi$ denotes the worst case page fault handling cost. Due to the assumption in the previous section, $\pi$ is the same in all cases. The demand paging cost for each execution path in task $\tau_{i}$ is: 


$$
\text { demand paging } \text { cost }_{i, j}=\pi \times n\left(P_{i, j}\right) .
$$

The WCET including demand paging cost for each execution path could be calculated as follows:

$$
C_{i}^{\prime \prime}=\max \left\{\begin{array}{c}
C_{i, 0}+\text { demand paging } \text { cost }_{i, 0} \\
C_{i, 1}+\text { demand paging } \text { cost }_{i, 1} \\
\ldots \\
C_{i, n-1}+\text { demand paging } \text { cost }_{i, n-1}
\end{array}\right\} .
$$

\subsection{Page Reuses Aspect Analysis}

The demand paging cost could be reduced among task instances since a task instance reuse the pages already loaded by preceding task instances. The objective of the page reuses aspect analysis is to obtain a safe bound on the demand paging cost considering the page reuses among task instances. For the analysis, we use graph-theoretical formulation that the execution time of each task instance (i.e., execution path) considering the page reload cost is represented. By traversing the graph from the start node until the last task instance to obtain the maximum distance, we obtain the WCRT of each task instance and the worst case scenario of task schedule. Figure 2(a) shows the overall page reuses aspect analysis in brief.

For the page reuses aspect analysis, we consider a directed graph $G=(N, E): N$ is the set of nodes that represent instances of each task and $E$ is the set of edges among the nodes whose weights are the execution times between two task instances. The number of elements of $N$ is the number of execution paths multiplied by the number of instances of each task. The weight of each edge, $w_{p, q, r}$, in $E$ represents the execution time of execution path $q$ of $r^{\prime}$ th task instance of when the immediately preceding path is $p$. For our formulation, we define $c_{p, q}$ as the WCET considering the demand paging cost of the execution path $q$ when the immediately preceding execution path was $p$. In addition, we define $\theta_{p, q, r}$ as the page reloads cost of the execution path $q$ for $r$ 'th task instance when the immediately preceding execution path was $p . w_{p, q, r}$ can be defined as $c_{p, q}-\theta_{p, q, r}$.

The page reuses aspect analysis is to determine the maximum sum of the WCETs considering the demand paging cost and page reuses for each task instances. The following formulation shows a problem definition.

$$
\text { Maximize } \sum_{r=1}^{n}\left(c_{p, q}-\theta_{p, q, r}\right) .
$$

where $(p, q) \in N$. Since the pure WCET of the task could not vary, $c_{p, q}$ is always the same in all cases. But $\theta_{p, q, r}$ ranges from 0 to the maximum page fault delay assuming that all the pages of execution path q cause faults. This is because the page reload cost varies according to the pages loaded in the previous task instances. 


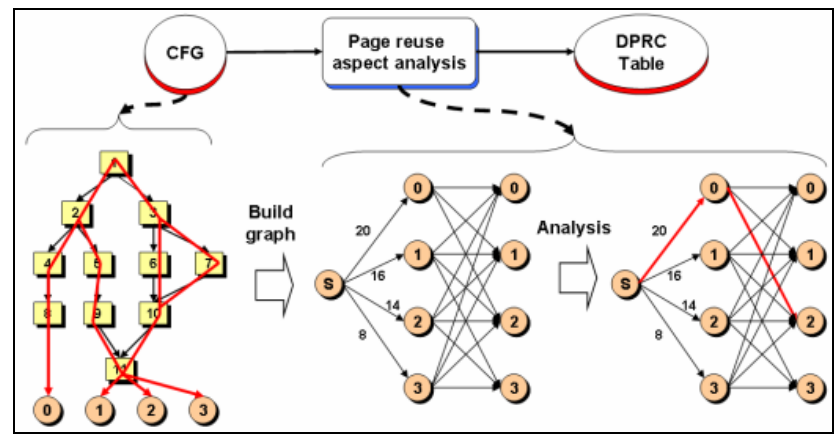

(a)

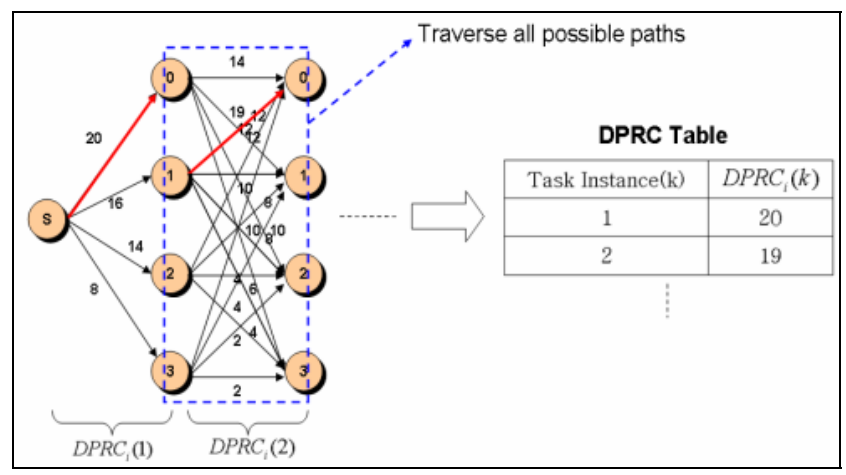

(b)

Fig. 2. The page reuses aspect analysis overview

The key point in the formulation is that in order to obtain the safe bound on the WCRT considering page fault cost, we choose the maximum weight for each pair of task instances. For describing the problem more formally, we introduce the following new notations, DPRC (Demand Paging Related Cost), and PLOAD.

$$
\begin{array}{ll}
\operatorname{DPRC} C_{i}(j) & \begin{array}{l}
: \text { the WCET of task instance } \tau_{i, j} \text { considering demand paging costs } \\
\text { and the page reuses }
\end{array} \\
\operatorname{PLOAD} D_{i}(j) & \begin{array}{l}
: \text { the set of all the combinations of pages loaded until task instance } \\
\tau_{i, j} .
\end{array}
\end{array}
$$

For example, the WCETs considering demand paging costs for task instances $\tau_{0,0}$, $\tau_{0,1}$ and $\tau_{0,2}$ are $D P R C_{0}(1), D P R C_{0}(2)$ and $D P R C_{0}(3)$, respectively. In the above graph, DPRC is the same as $c_{p, q}-\theta_{p, q, r}$ and PLOAD determines $\theta_{p, q, r}$.

Figure 2(b) shows how to calculate each DPRC values of the task. In the figure, the first task instance has the maximum WCET when the task executes an execution path 0 and in this case, $D P R C_{i}(1)$ is 20 . In order to calculate $D P R C_{i}(2)$, all the possibilities of page loads for the task instance $\tau_{i, 1}$ should be re-considered. As a result, 
$D P R C_{i}(2)$ is 19 when the task executes the execution path 0 . This would produce safe bound on the WCET of the second task instance.

\subsection{Calculation of the WCRT}

In this paper, we propose two different WCRT analysis methods: DP-Pessimistic and DP-Accurate, depending on the accuracy and the complexity of the analysis. In DPPessimistic method, the WCRT $R_{i}$ of task $\tau_{i}$ is calculated only by using the demand paging cost analysis and could be formulated as follows:

$$
R_{i}=C_{i}^{\prime \prime}+\sum_{j \in h p(i)}\left\lceil\frac{R_{i}}{T_{j}}\right\rceil C_{j}^{\prime \prime}
$$

where $C_{i}^{\prime \prime}$ is the new WCET including demand paging cost recalculated from $C_{i}$. In DP-Accurate method, the WCRT $R_{i}$ of task $\tau_{i}$ is calculated using the demand paging cost analysis and page reuses aspect analysis and could be obtained as follows:

$$
R_{i}=D P R C_{i}(1)+\sum_{j \in h p(i)} \sum_{k=1}^{\left\lceil\frac{R_{i}}{T_{j}}\right\rceil} D P R C_{j}(k) .
$$

where $D P R C_{i}(1)$ and $D P R C_{j}(k)$ are the WCETs of each task instance considering the demand paging cost and page reuses.

\section{Evaluations}

To evaluate the analysis accuracy of DP-Pessimistic and DP-Accurate, we simulate the task executions with demand paging using manually composed sample task set with various task parameters. Our simulator performs RM (rate monotonic) scheduling simulation with three different demand paging cost analysis: shadowing, DPPessimistic, and DP-Accurate. For our evaluation experiments, we carefully select the task parameters to show the effect of DP-accurate compared to DP-pessimistic as much as possible.

Table 1 shows the sample task set and parameters. Each task set contains same number of tasks with the same pure WCETs when demand paging costs are not considered. The only differences among the task sets are the page access behaviors of the tasks to show the accuracy differences of DP-Pessimistic and DP-Accurate methods. Task set 2 and Task set 3 have execution paths whose demand paging costs are significantly larger than the costs of other paths.

Figure 3 shows the WCRT analysis results for the lowest priority task of each task set. The execution times are given at $m s$ (milliseconds) and the ratio of DP-Accurate over DP-Pessimistic is given. The differences between DP-Accurate analysis and DPPessimistic are significant for task sets 2 and 3 since the task sets have execution paths whose page access costs are significantly larger than other paths. This reveals that DP-Pessimistic analysis produces too loose bounds on the WCRTs compared to DP-Accurate analysis. Such loose bound affects the decision on the schedulability of the task sets. For example, with DP-Accurate, all the task sets satisfy the deadlines, 
Table 1. Task set parameters

\begin{tabular}{|c|c|c|c|c|}
\hline Task set & $T_{i}$ & \# of paths & $C_{i, j}$ & $P_{i, j}$ \\
\hline \multirow{8}{*}{1} & 5 & 1 & 1 & $\{45\}$ \\
\hline & 15 & 1 & 2 & $\{46\}$ \\
\hline & \multirow{5}{*}{60} & \multirow{5}{*}{5} & 4 & $\{1,2\}$ \\
\hline & & & 3 & $\{8,9\}$ \\
\hline & & & 5 & $\{15,16\}$ \\
\hline & & & 4 & $\{24,25,26,27,28,29\}$ \\
\hline & & & 5 & $\{33,34,38,39\}$ \\
\hline & 240 & 1 & 60 & $\{47\}$ \\
\hline \multirow{8}{*}{2} & 5 & 1 & 1 & $\{45\}$ \\
\hline & 15 & 1 & 2 & $\{46\}$ \\
\hline & \multirow{5}{*}{60} & \multirow{5}{*}{5} & 4 & $\{1,2\}$ \\
\hline & & & 3 & $\{8,9\}$ \\
\hline & & & 5 & $\{15,16\}$ \\
\hline & & & 4 & $\{24,25,26,27,28,29,30,31\}$ \\
\hline & & & 5 & $\{38,39\}$ \\
\hline & 240 & 1 & 60 & $\{47\}$ \\
\hline \multirow{8}{*}{3} & 5 & 1 & 1 & $\{45\}$ \\
\hline & 15 & 1 & 2 & $\{46\}$ \\
\hline & \multirow{5}{*}{60} & \multirow{5}{*}{5} & 4 & $\{1,2\}$ \\
\hline & & & 3 & $\{8,9\}$ \\
\hline & & & 5 & $\{15,16\}$ \\
\hline & & & 4 & $\{24,25,26,27,28,29,30,31,32,33,34\}$ \\
\hline & & & 5 & $\{38,39\}$ \\
\hline & 240 & 1 & 60 & $\{47\}$ \\
\hline \multirow{8}{*}{4} & 5 & 1 & 1 & $\{45\}$ \\
\hline & 15 & 1 & 2 & $\{46\}$ \\
\hline & \multirow{5}{*}{60} & \multirow{6}{*}{5} & 4 & $\{1,2,3\}$ \\
\hline & & & 3 & $\{8,9,10\}$ \\
\hline & & & 5 & $\{15,16\}$ \\
\hline & & & 4 & $\{24,25,26,27\}$ \\
\hline & & & 5 & $\{38,39,40\}$ \\
\hline & 240 & & 60 & $\{47\}$ \\
\hline
\end{tabular}

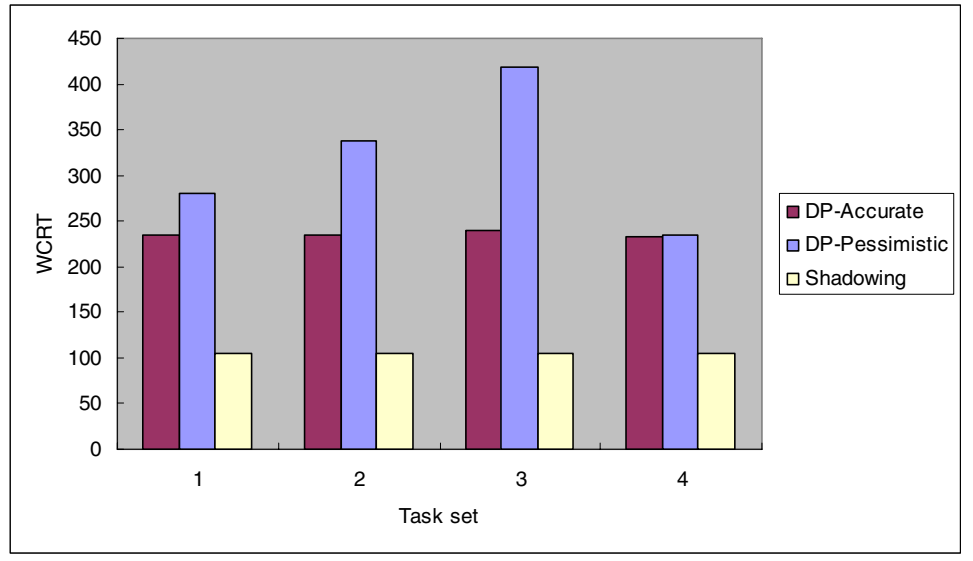

Fig. 3. Evaluation results 
but with DP-Pessimistic, the tasks for Task sets 1, 2, and 3 do not satisfy the deadlines from the analysis. Task set 4 shows similar worst case response times between DPAccurate and DP-Pessimistic since the page access costs are similar for all execution paths.

\section{Conclusion}

In this paper, we have proposed a WCRT analysis method considering demand paging costs. Depending on the accuracy and the complexity of the analysis, two different analysis methods, DP-Pessimistic and DP-Accurate, are devised. DP-Accurate considers the possibility of page reuses among different task instances while DPPessimistic does not consider the page reuses.

Evaluation results show that DP-Accurate method gives more accurate analysis results than DP-Pessimistic method especially for the tasks consisting of task instances whose pages are reused by succeeding task instances. The current analysis method is the basis for future extensions that aim at more practical modeling of demand-paging for real-time systems.

Acknowledgments. This work was supported in part by the MIC \& IITA under IT Leading R\&D Support Project 2006, in part by No. 379 from the Basic Research Program of KOSEF, in part by the MIC, Korea under the ITRC (Information Technology Research Center) support program supervised by the IITA (IITA-2006-C10900603-0045), and in part by Kookmin University under Research Center Incubation Program (UICRC).

\section{References}

1. K. Tindell.: Adding Time-Offsets to Schedulability Analysis. Technical Report YCS 221, Dept. of Computer Science, University of York, England (1994)

2. Palencia, J. C. and Gonzlez Harbour, M.: Schedulability Analysis for Tasks with Static and Dynamic Offsets. In Proceedings of the IEEE Real-Time Systems Symposium (1998)

3. N.Audsley et al.: Applying new scheduling theory to static priority preemptive scheduling. Software Engineering Journal. (1993) 284-292

4. J. V. Busquets-Mataix, J. J. Serrano-Martin, R. Ors, P. Gil, and A. Wellings.: Adding Instruction Cache Effect to Schedulability Analysis of Preemptive Real-Time Systems. Proceedings of the 2nd Real-Time Technology and Applications Symposium (1996)

5. C. Lee, J. Hahn, Y. Seo, S. Min, R. Ha, S. Hong, C. Park, M. Lee, and C. Kim.: Analysis of Cache-related Preemption Delay in Fixed-Priority Preemptive Scheduling. IEEE Transactions on Software Engineering (1996) 264-274

6. K. Tindell, A. Burns, and A. Wellings.: An Extendible Approach for Analysing FixedPriority Hard Real-Time Tasks. Journal of Real-Time Systems, 6(2). (1994) 133-151

7. Scott F. Kaplan, Lyle A. McGeoch, Megan F. Cole.: Adaptive caching for demand prepaging. Proceedings of the 3rd international symposium on Memory management. Berlin, Germany (2002) 